\title{
Effect of Curcumin and Vitamin D3 on Learning and Cognition Inrat Model of Alzheimer's Disease
}

\author{
Khan $\mathrm{S}^{1 *}$, Mas Rina Wait Hj. Abdul Hamid ${ }^{1}$ and \\ Kaneez FS ${ }^{2}$ \\ ${ }^{1}$ PAP Rashidah Sa'adatul Bolkiah Institute of Health \\ Sciences, Universiti Brunei Darussalam, Brunei \\ ${ }^{2}$ School of Medicine and Molecular Biosciences, \\ University Technology Sydney, Australia \\ *Corresponding author: Saima Khan, PAP Rashidah \\ Sa'adatul Bolkiah Institute of Health Sciences, Universiti \\ Brunei Darussalam, J alanTungku Link, BE1410 Negara, \\ Brunei
}

Received: April 04, 2017; Accepted: May 23, 2017; Published: J une 07, 2017

\begin{abstract}
Alzheimer's disease (AD) is a progressive neurodegenerative disorder due to gradual memory loss and shrinkage of neuronal cells particularly in the hippocampus and basal forebrain regions. The aim of this study is todeterminethe effect of Curcumin and Vitamin D3 for improving AD care and to design strategy for treatment and prevention of $A D$. Curcumin and vitamin $D 3$ used as neuroprotective agents against scopolamine-induced dementia in male Sprague-Dawleyrats. Scopolamineinduced impaired cognition was observed with behavioral tasks including; rectangular maze test and locomotor activity. In scopolamine treated animals, the correct response rate during acquisition and retention period was significantly lower than the control group. Curcumin and vitamin D3 showed improvement in cognition and learning. The correct response rate for both tasks was significantly equal to the control group $(p<0.05)$. $\mathrm{H}$ and $\mathrm{E}$ staining of rat brain scopolamine group showed less number of cells. Curcumin and vitamin D3 groups showed significantly increased number of cells compared with the scopolamine group. No gap around neuronal cells was observed in the drug treated groups compared with scopolamine group. Immunoblotting results demonstrated significantly presence of abnormal protein in scopolamine group compared with drug-treated groups. The presence of abnormal protein could be the reason of memory loss event in the rat brain observed during the behavioral study of scopolamine group compared with the control group. We can conclude that Curcumin and vitamin D3 may have potential to reverse some cognitive deficits and protect brain from cell degeneration.
\end{abstract}

Keywords: Alzheimer's disease; Memory impairment; Inflammation; Scopolamine; Curcumin; Vitamin D3 and donepezil

\section{Introduction}

Alzheimer's disease $(\mathrm{AD})$ is a neurodegenerative disorder of the elderly population. It is characterized by progressive loss of cognitive functions, amyloid $\beta(A \beta)$ deposition and formation of neurofibrillary tangles (NFTs) in the brain cells. NFTs are formed inside the cell bodies of the neurons. These NFTs cause shrinkage of neurons and this process would be a reason for theloss of cognition and teach [1]. Oxidative stress is considerably important for $\mathrm{AD}$ pathology. It is characterized by oxidization of the lipid, protein and nucleic acid components of the cell, inflammation, and $A \beta$ deposition [2]. Low central cholinergic levels and loss of cholinergic neurons have been linked with the key events in $\mathrm{AD}$ [3]. The earliest degeneration occurs in basal forebrain cholinergic nuclei found at pre dementia stages [4]. The decline of cholinergic transmission is also found to be associated with increased amyloid formation [5]. Similarly, tau hyperphosphorylation can be the earliest event in abnormal processing of tau protein during $\mathrm{AD}$ pathogenesis [6]. Hyperphosphorylated tau involves in synaptic dysfunction specifically in long-term depression (LTD) [7].

Animal models have proved that loss of synaptic plasticity is the key components in the neurodegenerative process of $\mathrm{AD}$ and tau is one of contributing factors for neurodegeneration [8]. In this study neuroprotective effects of Curcumin and vitamin D3, on scopolamine-induced learning and memory impairment in animals are investigated. Scopolamine is one of a group of drugs which acts on muscarinic acetylcholine receptors. It is widely used in experimental animals.

\section{Role of curcumin in alzheimer's disease}

Alzheimer's patients that have revealed that $A \beta$ plaques are surrounded by active microglia and astrocytes and this activated microglia secrete proinflammatory and some reactive substances that deteriorate $A \beta$ pathology. Curcumin exerts its anti- proliferative action on microglia due to the ability to pass through all the cell membranes. Its minimal dose can distress neuroglial proliferation and differentiation [9]. It is beneficial to improve behavioral disturbance in an animal model of $\mathrm{AD}$ [10]. Curcumin has proved significant improvement in behavioral symptoms when AD patients were given turmeric treatment [11]. Curcumin has ability to enter in the brain and reduced toxicity a study published in the journal of Alzheimer's disease (News.vanderbilt.edu/2015/01/08/curcumin'sability-to-fight-Alzheimer-studied/). While this news also published that use of aerosolized Curcumin is more beneficial to cortex and hippocampus than intravenous injection in a transgenic mouse model of Alzheimer's disease," Pham said.

\section{Role of vitamin D3 in alzheimer's disease}

Vitamin D3 acts as a neuroprotective agent through endogenous hormonal form $1 \alpha, 25$ dihydroxyvitamin $\mathrm{D}$, it has a high potential for prevention of $\mathrm{AD}$. It acts directly and indirectly in neurons by 
improving the clearance of $A \beta$ by macrophages/microglia $[12,13]$. Vitamin D has multiple biological targets can be used as an adjunct to standard anti-dementia treatments for corrections of neurological deficits in $\mathrm{AD}$.

\section{Materials and Methods}

\section{Animals}

Male Sprague-Dawley rats of $200 \pm 25 \mathrm{~g}$ were obtained from the animal house (University Brunei Darussalam). They were housed individually in a cage (at an ambient temperature of $25 \pm 2^{\circ} \mathrm{C}$ ) and 45 $55 \%$ relative humidity with 12 hours light/dark cycles. Rats were free to access food and water ad libitum.

All rats were handled daily for a minimum of 1 week prior to behavioral testing. One week prior to testing in the "Rectangular Maze" and throughout testing in the rectangular Maze and for locomotor activity using "Actophotometer", the food was restricted to a daily feeding of approximately $80 \%$ of their ad libitum consumption to maintain the weight of each rat at approximately its freely fed weight. Experiments were performed between 09:00 am to 5:00 pm to reduce the stress effect of noise and other variants. A constant temperature of $25 \pm 2{ }^{\circ} \mathrm{C}$ and 12:12 light-dark cycle were maintained throughout the experiments.

\section{Experimental design}

- All experiments were conducted during the daytime between 9:00 am to 5:00 $\mathrm{pm}$.

- The procedure of drug treatment was carried out for 27 days.

- All experiments were conducted in accordance with an institutional guideline for animal care and use.

\section{Drug treatments}

There were 30 animals divided into 5 groups of six animals in each group. All groups except vehicle group received scopolamine ( $2.5 \mathrm{mg} / \mathrm{kg}$ body wt.) i.p. injection for each day for 27 days to induce excitotoxicity. Donepezil $(2.5 \mathrm{mg} / \mathrm{kg}$ ) (serves as a standard drug) oral dose was given to experimental groups for each day for 27 days. Group 5 received vitamin D3 $(0.0179 \mathrm{mg} / \mathrm{kg})$ oral for days for 27 days. Curcumin $(80 \mathrm{mg} / \mathrm{kg})$ oral dose was given to experimental groups except for vehicle group. Vehicle group was given $0.9 \%$ saline (i.p. Injection).

\section{Behavioral tests}

All groups of animals were given training for behavioral tests for one week before drugs induction.

Rectangular maze test: Learning, memory, and reasoning in animals were assessed using rectangular maze. The animal with the intact memory makes use its past experience, to find a way out in the maze [14]. Rectangular maze it is a rectangular box with the entry and reward (food) appended at opposite ends. Each animal was trained prior to drug induction with the maze. Time was taken to the animal to reached reward chamber was recorded. For each animal, five reading are taken and the average is calculated as their learning score [15].

Locomotor activity: CNS drugs have an influence on locomotor activity in both man and animals. The equipment measures the locomotor activity of drug operates on photoelectric cells which are connected in circuit with a counter. When the ray of light falls on photocells is cut off by the animal then, a count is recorded [16]. Each animal has given 2 minutes inactivity cage and activity is recorded. The count is recorded and increase or decrease in locomotor activity was calculated.

Histology: After a behavioral study on animals, they were anesthetized and decapitated. Brains were removed from the skull and stored in paraformaldehyde (4\%), and then brains were embedded in paraffin and kept in there frigerator. $5 \mu \mathrm{m}$ coronal sections were prepared using rotary microtome stained with Hematoxylin and Eosin. Photographs were taken for each section [17]

\section{Estimation of protein concentration:}

Immunoblotting: Antibodies against modified microtubuleassociated protein Tau was obtained from Thermo scientific. Dissect the brain tissue of coronal area with clean tools on ice, and as quickly as possible to prevent degradation by proteases. Placed the tissue in micro centrifuge tubes and immerse in liquid nitrogen to snap freeze. Homogenized brain tissues on the ice $1 \mathrm{X}$ ice-cold lysis buffer rapidly added to the tube, homogenized with an electric homogenizer, rinsed twice a rinsed twice with the same conc. of lysis buffer, and agitation was maintained for 2 hours at $4^{\circ} \mathrm{C}$. Centrifugation was done for 20 min at $12000 \mathrm{rpm}$ at $4{ }^{\circ} \mathrm{C}$ micro centrifuge tube was then placed on ice. The supernatant was removed and placed in a fresh tube kept on ice; discard the pellet. A small volume of lysate was taken to perform a protein quantification assay.

Boil each cell lysate in TBST buffer at $100{ }^{\circ} \mathrm{C}$ for $5 \mathrm{~min}$. 50 micrograms of protein were loaded into the wells of the SDS-PAGE gel. Gal was run for 1-2 hours at $100 \mathrm{~V}$. Transferred the protein onto the membrane. The membrane was blocked for 1 hour at room temperature. Membrane washed with TBST 3 times 5 min each. The membrane was incubated with secondary antibody in blocking buffer at room temperature for 1 hour. It was washed with TBST buffer three times $5 \mathrm{~min}$.

Blocked the membrane for 1 hour at room temperature. The membrane was then incubated with 1:1000 dilution of primary antibody in blocking buffer. Washed the membrane in three washes of TBST, 5 min each. Incubated the membrane with the 1:1000 dilution of conjugated secondary antibody in blocking buffer at room temperature for 1 hour. Wash the membrane in three washes of TBST, 5 min each. Remove excess reagent and cover the membrane with thetransparentplastic wrap. Acquired image using darkroom development techniques for chemiluminescence detection.

\section{Results \\ Behavioral tests}

Rectangular maze test: The neuroprotective activity of curcumin vitamin D3 was evaluated using a rectangular maze. Donepezil was used as standard drug. The rats in Curcumin and vitamin D3 groups except for scopolamine-treated group (disease control group) showed lower transfer latency (time taken by the animal to move from open arm to coverd arm) in Figure 1. The performanceof rats between groups (control, scopolamine and drug-treated groups) significant 


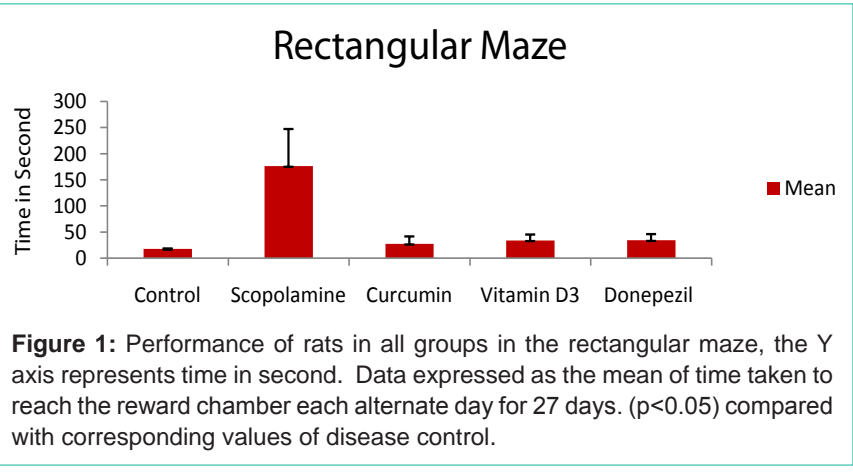

Table 1: Drug treatment schedule.

\begin{tabular}{|c|c|c|}
\hline Group I & $\begin{array}{l}\text { Saline- } \\
\text { control }\end{array}$ & ( $0.9 \%$ saline $)+$ behavioral test \\
\hline Group II & $\begin{array}{l}\text { Disease } \\
\text { control }\end{array}$ & Scopolamine $(2.5 \mathrm{mg} / \mathrm{kg})$ i. p. + behavioral test \\
\hline $\begin{array}{l}\text { Group } \\
\text { III }\end{array}$ & Experimental & $\begin{array}{c}\text { Scopolamine }(2.5 \mathrm{mg} / \mathrm{kg}) \text { i. p. Curcumin }(80 \mathrm{mg} / \mathrm{kg}) \text { oral } \\
+ \text { behavioral test }\end{array}$ \\
\hline $\begin{array}{l}\text { Group } \\
\text { IV }\end{array}$ & Experimental & $\begin{array}{c}\text { Scopolamine }(2.5 \mathrm{mg} / \mathrm{kg}) \text { i. p., Donepezil } \\
(2.5 \mathrm{mg} / \mathrm{kg}) \text { oral + behavioral test }\end{array}$ \\
\hline Group V & Experimental & $\begin{array}{l}\text { Scopolamine }(2.5 \mathrm{mg} / \mathrm{kg}) \text { i. } \mathrm{p} \text {. } \\
\text { Vitamin D3 }(0.0179 \mathrm{mg} / \mathrm{kg}) \text { oral +behavioral test }\end{array}$ \\
\hline
\end{tabular}

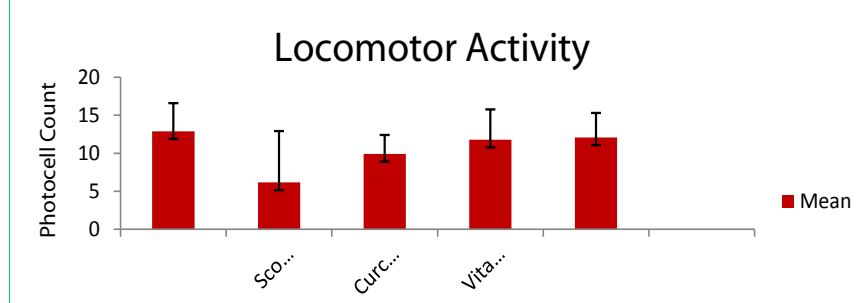

Figure 2: Represent locomotor activity of all rats except scopolamine treated rats in actophotometer showed no significant difference in drug treated groups. Data expressed as mean of photocell count $(n=6)$ of animals each alternate days for 27 days. $(p<0.05)$ compared with corresponding values of disease control.

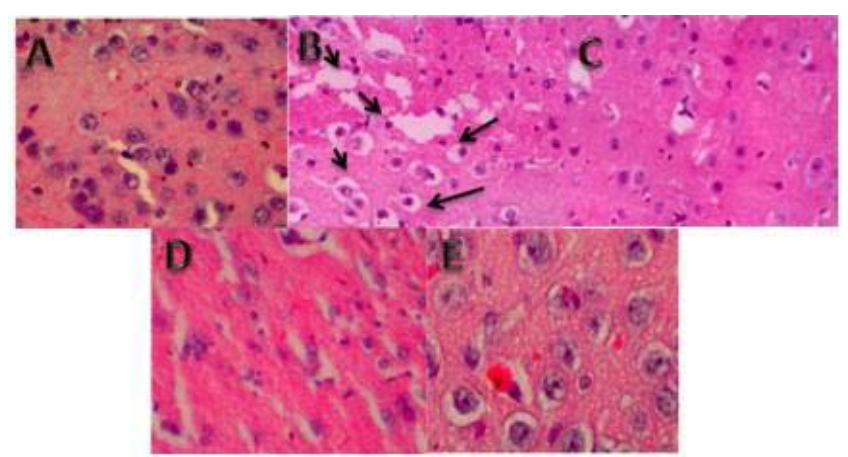

Figure 3: Hematoxylin and Eosin staining for A control, B Scopolamine, C Curcumin, D Vitamin D3 and E Donepezil. Images show no significant difference in cellular histology in the hippocampal area found in the experimental groups (Curcumin, Vitamin D3, and Donepezil) compared with the scopolamine group. Scopolamine group shows less number of nuclei stained with $\mathrm{H}$ and $\mathrm{E}$ staining. (Arrows in scopolamine slide $\mathrm{B}$ indicated the gaps around neuronal cells and in the slide) Coronal sections $(5 \mu \mathrm{m})$ at magnification $40 \mathrm{X}$.

difference across all days shown in the Figure $1(\mathrm{p}<0.05)$.

Locomotor activity: The locomotor activity of curcumin and

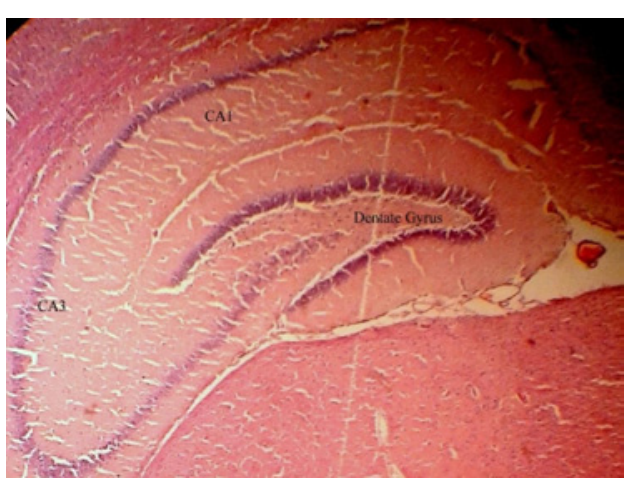

Figure 4: Coronal section of rat brain (Control group) at 10X to represent important memory keeping regions in the hippocampus of the brain.

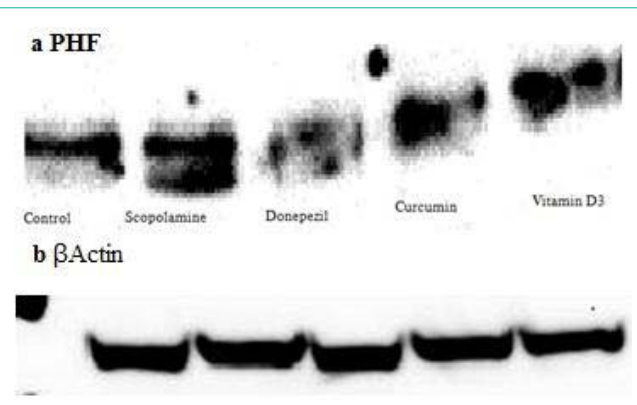

Figure 5: Western blot image. From left to right (Control, scopolamine, Donepezil, Curcumin+ scopolamine, Vitamin D3+scopolamine).

vitamin D3 treated rats was evaluated using actophotoactometer. The rats showed significant transfer latency in the experimental groups except for scopolamine treated group as shown in Figure 2. The performance of the groups (control, scopolamine and treated groups) showed a significant difference across all number of days. A significant difference was found in scopolamine group as shown in Table $1(\mathrm{p}<0.05)$.

Histology: It is shown in Figure 3, degeneration of cells is more prominent in the scopolamine group than in the drugs (curcumin and vitamin D3) treated groups. This degeneration was indicated by a gap arround the neuronal cells indicated by arrows and the slides after $\mathrm{H}$ and $\mathrm{E}$ staining. The drugs treated groups (Curcumin and vitamin D3) showed the almost equal number of cells and similar morphology of cells compared with control group. Figure 4 (Control group) represent important memory keeping regions in the hippocampus of the brain which was observed during histology study.

\section{Estimation of protein concentration:}

Immunoblotting: Curcumin and vitamin D3 reduce tau phosphorylation in the brains of as copolamine-treated rat model of Alzheimer's disease. (a) Immunoblot of hippocampus homogenates from treated rats (scopolamine, vehicle, treated with Curcumin and vitamin D3) using the PHF monoclonal antibodies. Figure 5 corresponds to the densitometric analysis of the bands normalized against $\beta$-Actin.

\section{Discussion}

In present study effects of Curcumin and vitamin D3 on an 


\section{Relativedensity}

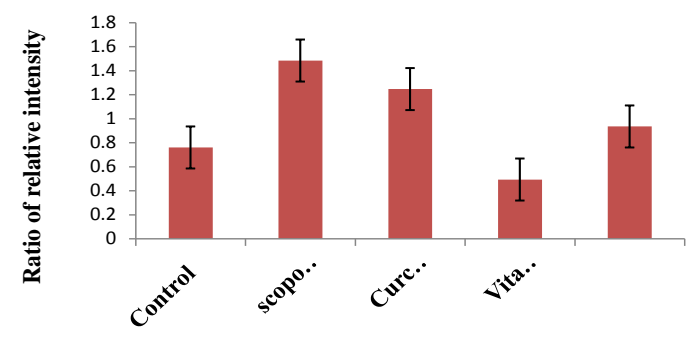

Figure 6: Densitometry data obtained and presented as the relative density of the tau protein present in all groups. It was normalized to $\beta$ actin protein.

animal model of $\mathrm{AD}$ was investigated. Scopolamine is widely used to make an animal model of AD for screening anti-Alzheimer's drug [18]. Scopolamine acts as muscarinic cholinergic antagonist causes impairment in cognition and learning.

Scopolamine amnesia has shown there was a general decrease in the transfer latency in all experimental groups as compared to the scopolamine-treated group from the behavioral study. The memory loss effect of scopolamine is more prominent compared to the control group. Studies have shown that use of NSAID treatment has dramatic effect on $\mathrm{AD}$ patients [19].

From behavioral test that is, rectangular maze test, and locomotor activity, it is clearly seen that there was a general decrease in the transfer latency in all treated groups compared to the scopolaminetreated group. The memory loss effect of scopolamine is more prominent compared to the control group. In comparison with Donepezil, the drug treated groups had almost equal performance against memory loss Figure 1 and 2.

The Curcumin and vitamin D3 treated group had almost equal performance compared with control group, which may indicative of the neuroprotective effect of Curcumin, against memory loss. To evaluate the effect of CNS drug such as scopolamine locomotor activity test was done on actophotometer which also indicates the improved learning ability in Curcumin and vitamin D3 treated group's Figure 1 and 2. On the other hand, histological studies showed no significant difference in all treated groups except scopolamine treated group. Scopolamine group has shown degenerated cells observed in Figure 6, there is a gap in the slide was seen when it is $\mathrm{H}$ and $\mathrm{E}$ stained. Scopolamine induced degeneration was clearly seen in previous studies [16]. While the Immunoblotting study of brain tissues using anti-human Paired Helical Filaments (PHF-Tau monoclonal antibody) (pdt.no.MN1020, thermo Scientific) have demonstrated significantly presence of abnormal Tau protein in disease control group when compared with treated groups. And the presence of abnormal Tau protein could be the reason of memory loss event in rat brain observed during the behavioral study of different groups. Therefore, on the basis of behavioral improvement in treated animals and Immunoblotting results, we can be concluded that Curcumin and vitamin D3 may have potential to reverse some cognitive deficits and memory impairment induced by scopolamine.

\section{Conclusion}

In conclusion, effects of Curcumin and vitamin D3 on learning and memory on an animal model of $\mathrm{AD}$ has shown improvement. The use of Curcumin and vitamin D3 can slow down the progress of $\mathrm{AD}$ pathologies or delay the onset of $\mathrm{AD}$ and therefore, could be used as a future drug for treatment of $\mathrm{AD}$. It indicates that both agents could be beneficial for the betterment of AD patients. Vitamin D due to its multiple biological targets sides can be used as an adjunct to standard anti-dementia treatments for corrections of neurological deficits in AD. Curcumin has intensively used for the betterment of $\mathrm{AD}$ symptoms; inhalable curcumin and ar-turmerone on neural stem cells (NSCs) investigations are in pipeline. However, further investigations on both compounds are needed to be carried out.

\section{References}

1. Brady S, Siegel G, Albers RW, Price D. Basic neurochemistry: molecular, cellular and medical aspects. 2005; Academic Press.

2. Chen Z, Zhong C. Oxidative stress in Alzheimer's disease. Neuroscience bulletin. 2014; 30: 271-281.

3. Mesulam M. The cholinergic lesion of Alzheimer's disease: a pivotal factor or side show?. Learning \& Memory. 2004; 11: 43-49.

4. Sassin I, Schultz C, Thal DR, Rüb U, Arai K, Braak E, et al. Evolution of Alzheimer's disease-related cytoskeletal changes in the basal nucleus of Meynert. Acta Neuropathol. 2000; 100: 259-269.

5. Beach TG. Physiologic origins of age-related ß-amyloid deposition. Neurodegenerative diseases. 2008; 5: 143-145.

6. Mondragon-Rodriguez S, Basurto-Islas G, Santa-Maria I, Mena R, Binder LI, Avila J, Smith MA, et al. Cleavage and conformational changes of tau protein follow phosphorylation during Alzheimer's disease. Int J Exp Pathol. 2008; 89: 81-90.

7. Mondragon-Rodriguez S, Perry G, Zhu X, Moreira PI, Acevedo-Aquino MC, Williams S. Phosphorylation of tau protein as the link between oxidative stress, mitochondrial dysfunction, and connectivity failure: Implications for Alzheimer's disease. Oxid Med Cell Longev. 2013; 2013.

8. Crimins JL, Pooler A, Polydoro M, Luebke JI, Spires-Jones TL. The intersection of amyloid beta and tau in glutamatergic synaptic dysfunction and collapse in Alzheimer's disease. Ageing Res Rev. 2013; 12: 757-763.

9. Mishra S, Palanivelu K. The effect of curcumin (turmeric) on Alzheimer's disease: An overview. Ann Indian Acad Neurol. 2008; 11: 13-19.

10. Ono K, Hasegawa K, Naiki H, Yamada M. Curcumin has potent antiamyloidogenic effects for Alzheimer's ß-amyloid fibrils in vitro. J Neurosci Res. 2004; 75: 742-750.

11. Hishikawa N, Takahashi Y, Amakusa Y, Tanno Y, Tuji Y, Niwa H, et al. Effects of turmeric on Alzheimer's disease with behavioral and psychological symptoms of dementia. AYU. 2012; 33: 499.

12. Fiala M. Re-balancing of inflammation and $A ß$ immunity as a therapeutic for Alzheimer's disease-view from the bedside. CNS Neurol Disord Drug Targets. 2010; 9: 192-196.

13. Rechel Champeau. Vitamin D, curcumin may help clear amyloid plaques found in Alzheimer's Early research findings may lead to new treatments for the disease. UCLA Newsroom. 2009.

14. Swain T. Effect of Galaolactoe on Learnigad Memory: A Study o role of Seroton in Pharmacol. online 2010; 3: 102-111.

15. Indumathy S, Kavimani S, Raman KV. The role of angiotensin antagonists in memory enhancement. International Journal of Pharma and Bio Sciences. 2010; 1: 1-4.

16. Goverdhan P, Sravanthi A, Mamatha T. Neuroprotective effects of meloxicam and selegiline in scopolamine-induced cognitive impairment and oxidative stress. International Journal of Alzheimer's Disease. 2012; 2012.

17. Jahanshahi M, Golalipour MJ, Afshar M. The effect of Urtica dioica extracts on the number of astrocytes in the dentate gyrus of diabetic rats. Folia Morphol (Warsz).2009; 68: 93-97. 
18. Kanwal A, Mehla J, Kuncha M, Naidu VGM, Gupta YKs, Sistla R. The antiamnesic activity of Vitex negundo in scopolamine-induced amnesia in rats. Pharmacology \& Pharmacy. 2010; 1: 1-8.
19. Stewart WF, Kawas C, Corrada M, Metter EJ. Risk of Alzheimer's disease and duration of NSAID use. Neurology. 1997; 48: 626-632.
Austin J Cerebrovasc Dis \& Stroke - Volume 4 Issue 3 - 2017

ISSN : 2381-9103 | www.austinpublishing group.com

Khan et al. () All rights are reserved
Citation: Khan S, Mas Rina Wait Hj. Abdul Hamid and Kaneez FS. Effect of Curcumin and Vitamin D3 on Learning and Cognition Inrat Model of Alzheimer's Disease. Austin J Cerebrovasc Dis \& Stroke. 2017; 4(3): 1060. 